\title{
Coercion and undue influence in decisions to participate in psychiatric research - methodological issues Robert Klitzman
}

\author{
Address: Columbia University: College of Physicians \& Surgeons, and School of Public Health; Ethics, Policy and Human Rights Core, HIV Center, \\ 1051 Riverside Drive/Unit \# 15, New York, New York 10032, USA \\ from WPA Thematic Conference. Coercive Treatment in Psychiatry: A Comprehensive Review \\ Dresden, Germany. 6-8 June 2007 \\ Published: 19 December 2007
}

BMC Psychiatry 2007, 7(SuppI I):SI38 doi:I0.II86/I47I-244X-7-SI-SI38

This abstract is available from: http://www.biomedcentral.com/I47I-244X/7/SI/SI 38

(c) 2007 Klitzman; licensee BioMed Central Ltd.

We are currently developing and testing an instrument that can potentially open up voluntariness to further study and reasoned policy-making. To substantiate the necessity and usefulness of efforts to protect the voluntariness of consent requires a valid and reliable way of assessing such voluntariness. Relatedly, e.g., the development of just such an instrument has opened up research on decisional competence, permitting more informed policy discussions. Presently, no reliable and valid measure of voluntariness exists. A few studies have examined, sometimes based on as a little as a single question, subjects' perceptions of whether they felt free to make participation decisions or would face sanctions for refusal. Yet no two studies have used the same methods, or reliable and valid instruments. Challenges arise in developing a measure: subjects' experiences and perceptions may not fully correlate with objective independent assessments, and constructs (e.g., "pressures") can be hard to operationalize. We are conducting open-ended interviews with researchers and subjects to identify reasons subjects offer for participation, determinants that may shape their choices (e.g., offers, pressures, and threats), and variations across populations. We are then drafting an instrument to assess determinants of subjects' decisions, and will be piloting it, demonstrating reliability and validity with subjects in clinical trials in 3 areas: substance abuse, HIV, and metastatic cancer, determining test-retest reliability, and convergent validity using 2 existing measures. We will discuss results from initial interviews with recruiters that suggest that, e.g., monetary and other incentives, rather than 'altruism,' play major roles in subjects' decisions. To begin to explore these issues in these ways can potentially contribute substantially to understanding the issues involved. 